\title{
Attitudes of Healthcare Professionals toward Patient Safety in the Operating Room
}

\author{
Reyhaneh Niknejad ${ }^{1}$, Mohammad Akbari ${ }^{2}$, Maryam Bagheri ${ }^{3}$, Maryam Hashemi ${ }^{4}$, \\ Fatemeh Ghaedi Heidari ${ }^{5}, *$ Akram Aarabi $^{6}$
}

\begin{abstract}
Background \& Aims: Patient safety is considered to be an inherent element of healthcare quality. Unsafe attitudes of healthcare employees are a major issue for healthcare providers in promoting the quality of care, which is closely correlated with the occurrence of hospital errors and incidents. On the other hand, development of optimal patient safety is not possible without establishing safety attitudes in healthcare facilities. The present study aimed to investigate the attitudes of healthcare providers toward patient safety in the operating room in the teaching hospitals in Isfahan, Iran.

Materials \& Methods: This cross-sectional, descriptive-analytical study was conducted on 217 healthcare practitioners in the teaching hospitals in Isfahan, Iran in 2018. The research units were selected via quota sampling. Data were collected using the standard safety attitude questionnaire (SAQ). Data analysis was performed in SPSS version 18 using descriptive and inferential statistics (independent t-test and Pearson's correlation-coefficient).

Results: The mean score of the total safety attitude of the healthcare providers was $59.06 \pm 11.84$ (total: 100). The highest score belonged to the dimension of stress recognition, with the mean score of $79.93 \pm 17.46$, and the lowest score belonged to the dimension of working conditions, with the mean score of 48.01 \pm 18.07 . Accordingly, $49.3 \%$ of the participants described their patient safety attitude to be good/excellent, while $50.7 \%$ described their attitude to be average/poor.

Conclusion: According to the results, the average safety attitude was not ideal in the operating room personnel. Therefore, it is recommended that hospital managers and staff attempt to implement effective interventions in order to promote the culture and attitude of patient safety in the operating room personnel.
\end{abstract}

Keywords: Attitude, Patient Safety, Operating Room, Healthcare Providers

\section{Conflict of Interest: No}

How to Cite: Niknejad R, Akbari M, Bagheri M, Hashemi M, Ghaedi Heidari F, Aarabi A. Attitudes of Healthcare Professionals toward Patient Safety in the Operating Room. Iran Journal of Nursing. 2019; 32(117):80-90.

Received: 22 Jan 2019

Accepted: 22 Apr 2019

\footnotetext{
1. MS, Department of Operating Room, Student Research Committee, School of Nursing and Midwifery, Isfahan University of Medical Sciences, Isfahan, Iran

${ }^{2}$. PhD in Nursing, Student Research Committee, School of Nursing and Midwifery, Isfahan University of Medical Sciences, Isfahan, Iran

${ }^{3}$. PhD in Nursing, Student Research Committee, Department of Adult Health Nursing, School of Nursing and Midwifery, Isfahan University of Medical Sciences, Isfahan, Iran

${ }^{4}$. PhD in Nursing, Department of Critical Care Nursing, Faculty of Nursing and Midwifery, Nursing and Midwifery Care Research Center, Isfahan University of Medical Sciences, Isfahan, Iran

5. PhD student in Nursing, Student Research Committee, School of Nursing and Midwifery, Isfahan University of Medical Sciences, Isfahan, Iran

6. Assistant Professor, Nursing and Midwifery Care Research Center, School of Nursing and Midwifery, Isfahan University of Medical Sciences, Isfahan, Iran (*Corresponding author)

Tel: 03137927510
}

Email: aarabi@nm.mui.ac.ir 


\title{
نكرش مراقبين سلامت نسبت به ايمنى بيمار در اتاق عمل
}

\author{
ريحانه نيك نزاد'، محمد اكبرى ؟، مريم باقرى 'ّ، مريم هاشمى
}

زمينه و هدف: ايمنى بيمار يكى از اجزاى مهم در كيفيت مراقبتهاى بهداشتى محسوب مىشود. يكى از مشكلات اصلى ارائه دهندكان خدمات

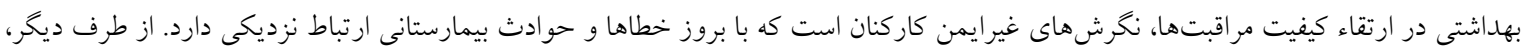

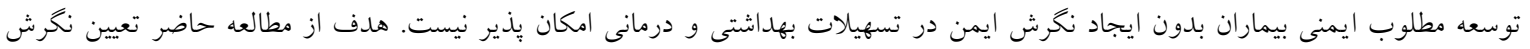

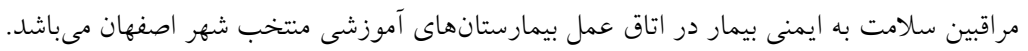
روش بررسى: اين يزوهش يك مطالعه مقطعى ازنوع توصيفى- تحليلى است كه در سال IT IV بر روى TIV نقر از مراقبين سلامت شاغل دراتاق

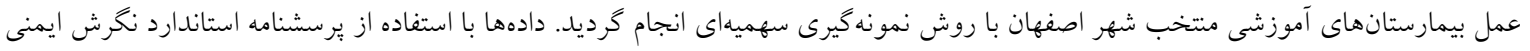

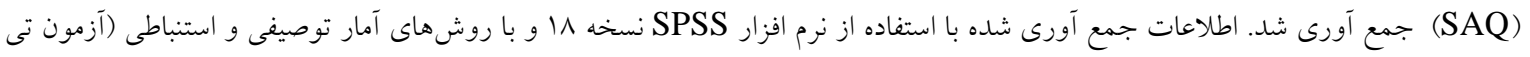

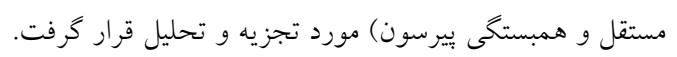

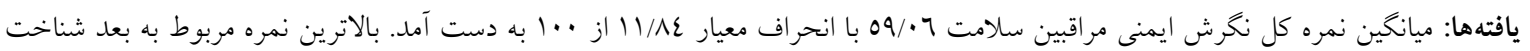

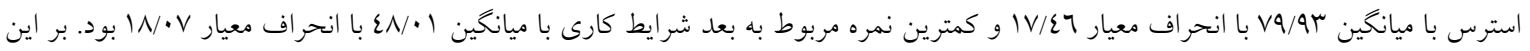

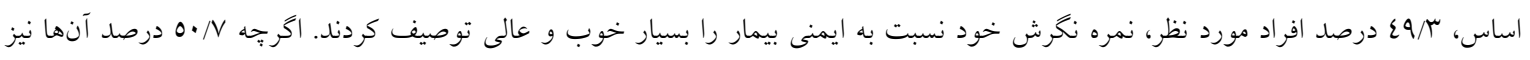
اين نمره ايمنى را متوسط تا ضعيف ذكر كردند.

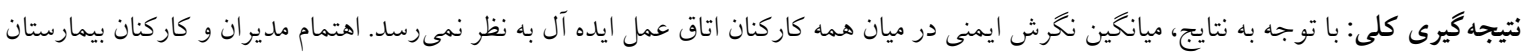

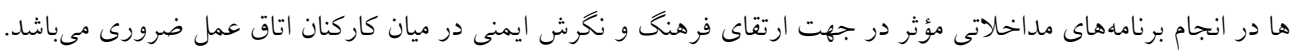
كليدوازهها: نخرش، ايمنى بيمار، اتاق عمل، مراقبين سلامت تعارض منافع: ندارد 9V/II/T: تاريخ دريافت تاريخ بذيرش:

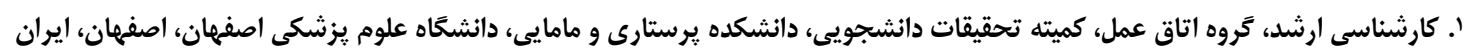

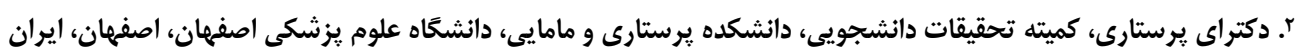

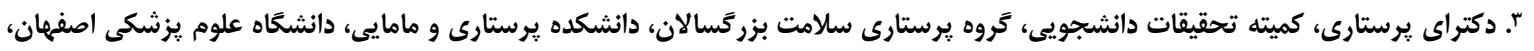
اصفهان، ايران إن دكتران

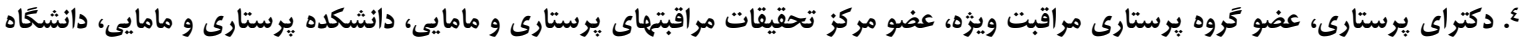
علوم يزشكى اصفهان،اصفهان، ايران

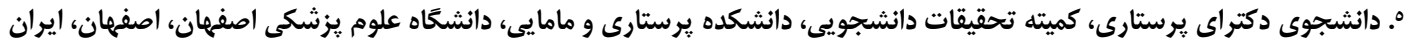

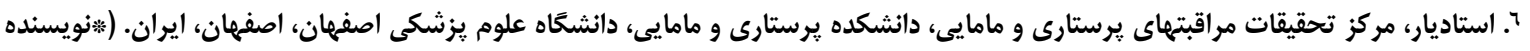


دادند. نتايج اين مطالعه مشخص نمود كه بيشتر كاركنان مقدمه نمره نخرشى در حد متوسط دارند (19). تورانى و همكاران در سال 90با نخرش نسبت به ايمنى بيمار را از ديدكاه

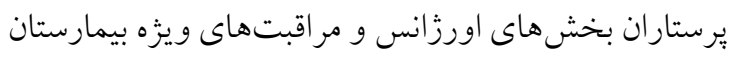

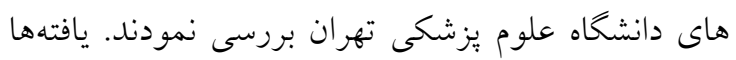

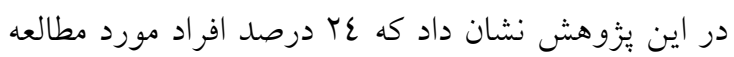
نمره ايمنى ضعيف و نامناسب، 2 درصد نمره ايمنى قابل

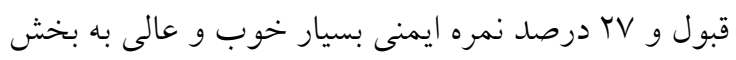
شاغل در آن دادند (Tr). يكى از بيبحيدهترين محيطهاى كار در نظام مراقبتهاى داى

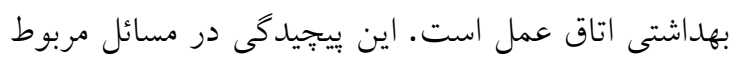

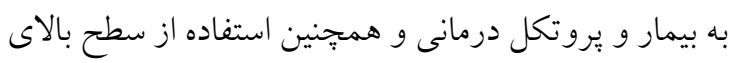

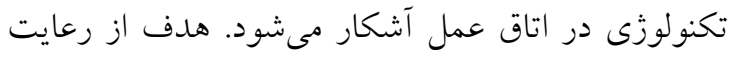
نكات ايمنى در اتاق عمل، تأمين ايمنى كاركنان شاغل در درّل

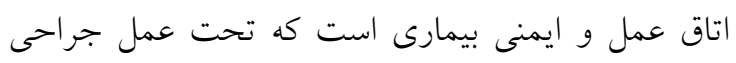

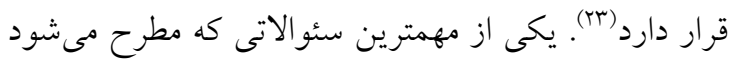

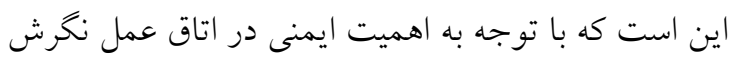

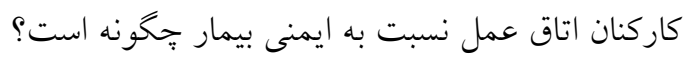

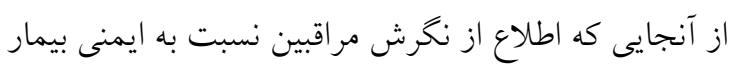
راه را براى برنامه ريزى در جهت افز ايش ائ ايمنى بيماران

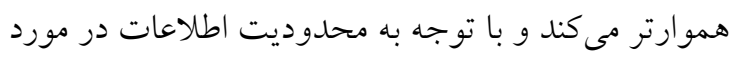

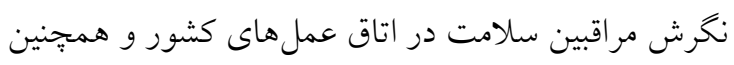

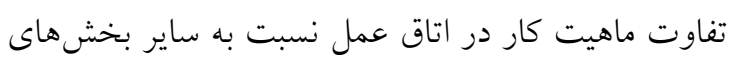

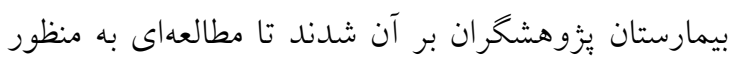

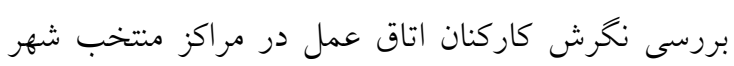
اصفهان انجام دهند.

\section{روش بررسى}

يزوهش حاضر يك مطالعه توصيفى از نوع مقطعى است بت بروست كه به منظور بررسى نكرش مر اقبين سلامت به ايمنى بيمار

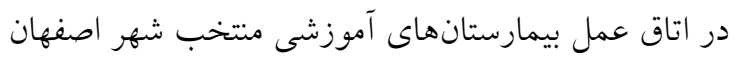

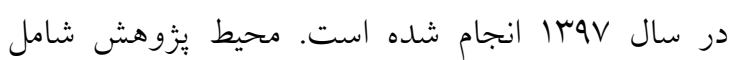
اتاقهاى عمل نه مركز آموزشى - درمانى وابسته به دانشگاه علوم يزشكى اصفهان و جامعه يُزوهش را تمامى مر اقبين مسأله ايمنى بيمار يكى از مهمترين مسائل مورد توجه در

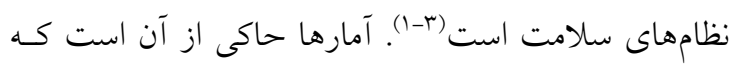

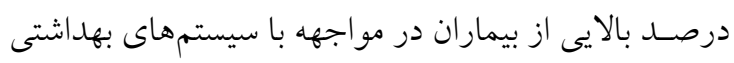

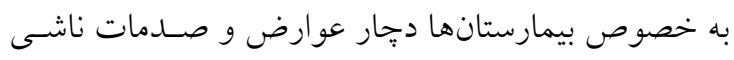
از ارائه خدمات مى گردند و مشكلى بر مشكلات اوليه آن بـان

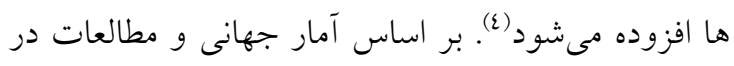

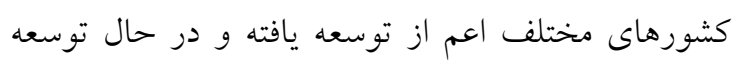

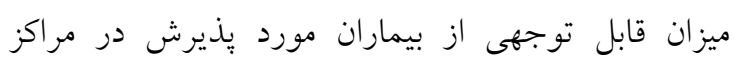

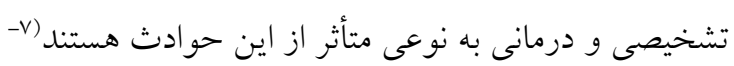

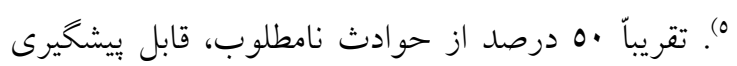

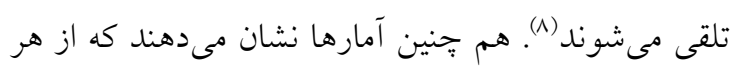

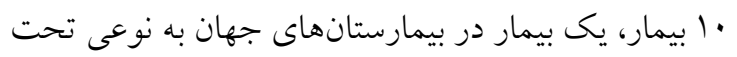

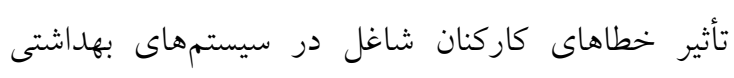

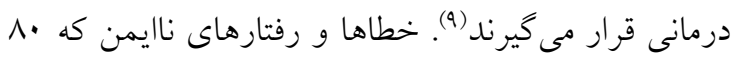

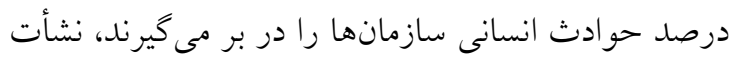

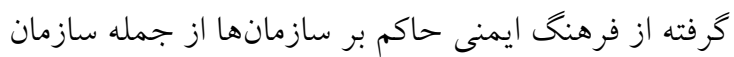
هاى ارائه دهنده خدمات سلامتى هستند (•).

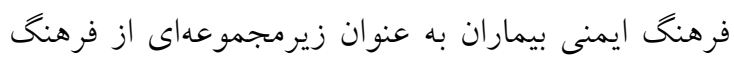

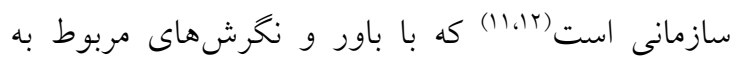

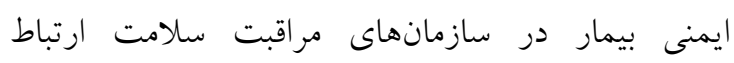

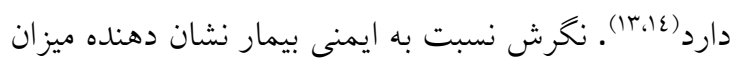

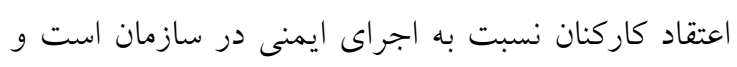
ارزيابى و شناخت مشكلات آن، به عنوان يكى هشدار اوليك درنيه

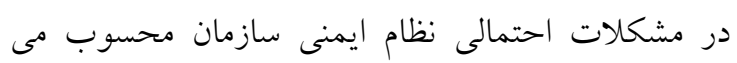

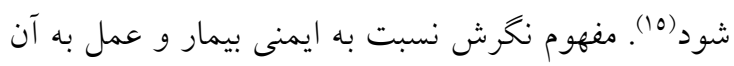

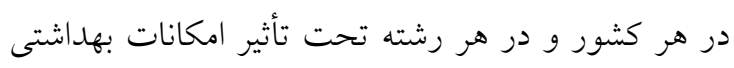

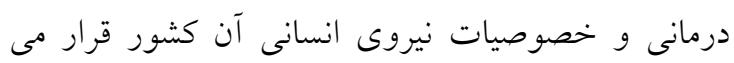

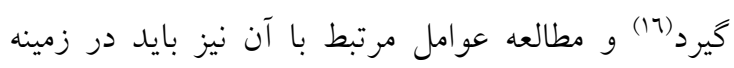
فرهنگى و شرايط سازمانى آن جامعه انجام يذيرد (IV).

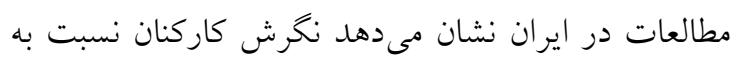

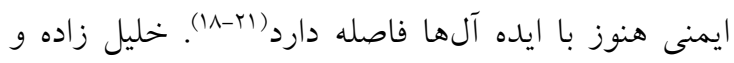

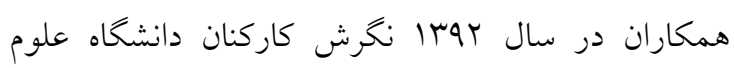
يزشكى اروميه را نسبت به ايمنى بيمار مورد بررسى قرار 
سابقه كار مراقبين سلامت مورد بررسى قرار كرفت و در قسمت دوم، دادهها با استفاده از يرسشنامه استاندارد نحرش ايمنى (SAQ) كه توسط Ban Sexton ساخته شده(r) و روايى و پِايايى آن قبلاً مورد تأييد قرار

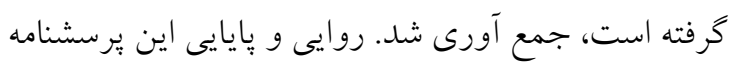

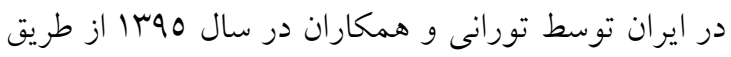

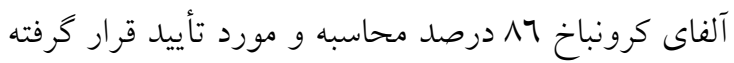

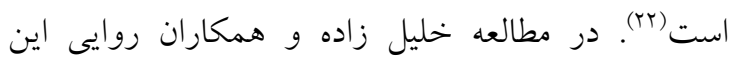
برسشنامه از طريق روايى محتوى و بايايى آن با آزمون

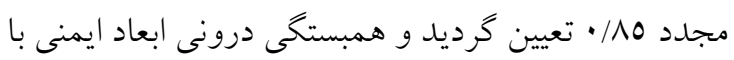

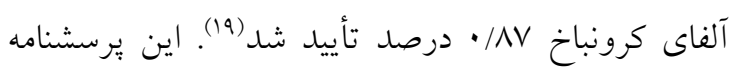

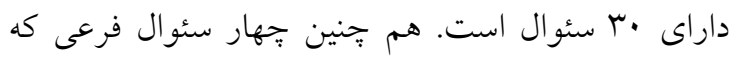

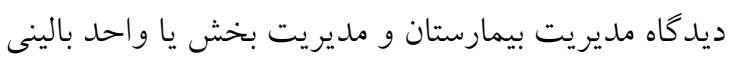

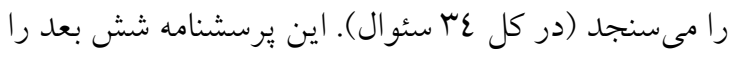

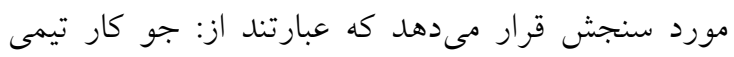

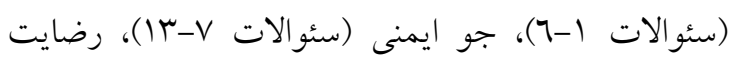

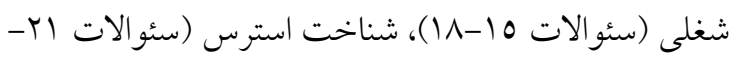
19)، نخرش نسبت به حمايت مديريت بيمارستان و بخش

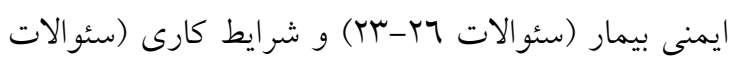

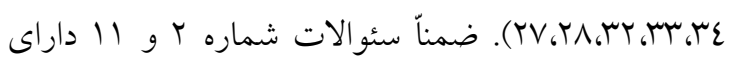

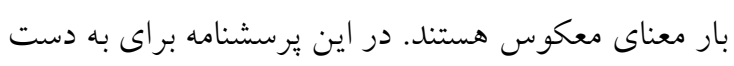

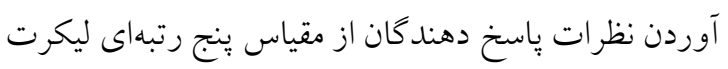

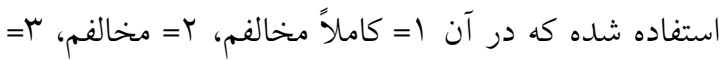

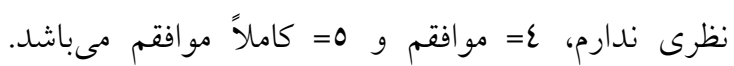

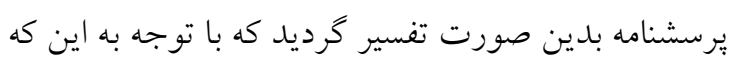

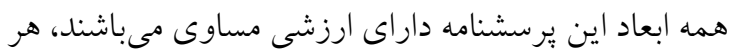

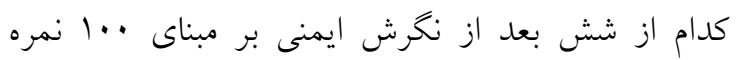

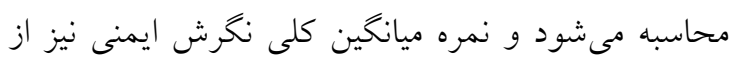
صفر تا .إ رتبه بندى مى موده، به طورى كه نمره كل

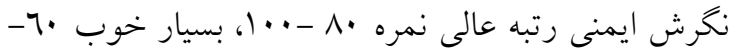

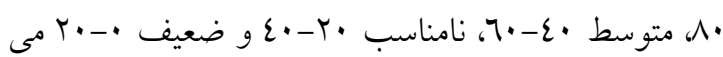

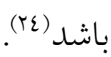
محقق پِس از دريافت مجوزهاى لازم از دانشكده يرستارى

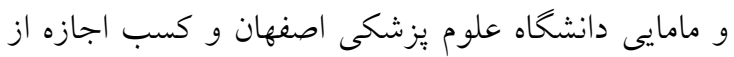

سلامت شامل جراحان، متخصصان بيهوشى، دستياران، تكنولوزيستها (كارشناسان و كارشناس ارشدهاى اتاق

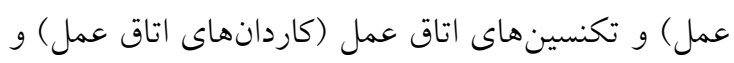
بيهوشى شاغل در اتاق عمل بيمارستانهاى مذكور تشكيل

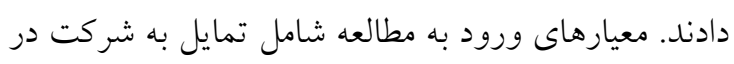

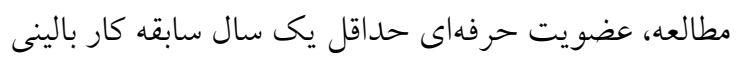
در اتاق عمل، حداقل مدرك تحصيلى كاردانى و شركت نكردن همزمان در مطالعه مشابه ديخر در يكى ماه اخير بود.

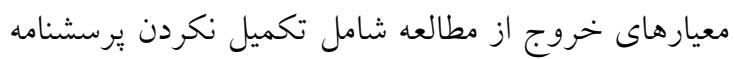

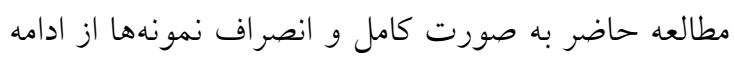

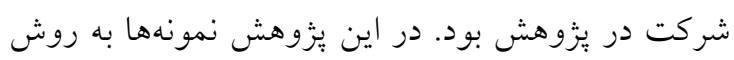
تخصيص متناسب از نوع سهميهاى و طبقهاى انتخاب شدند. در تخصيص سهميهاى ابتدا تعداد كل مراقبين سلامت در اتاقهاى عمل هر مركز درمانى مشخص شدندئ.

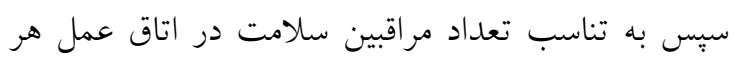

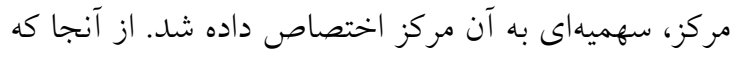
مراقبين سلامت شامل جندين طبقه بودند، در نمونه كيرى طبقهاى، سهميه اختصاص داده شده به اتاق عمل هر مركز درمانى، در طبقات مختلف به نسبت تعداد اعضاى حرفه

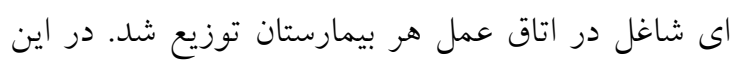

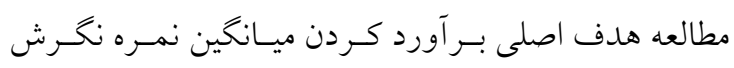

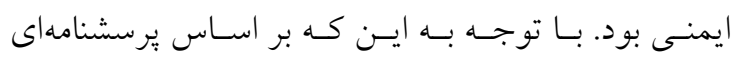

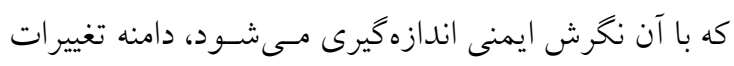

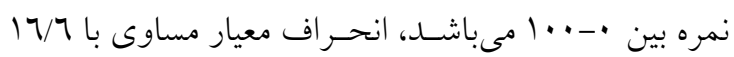

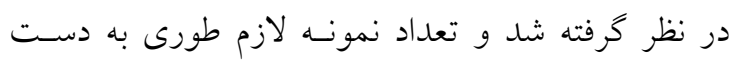

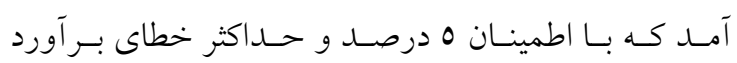

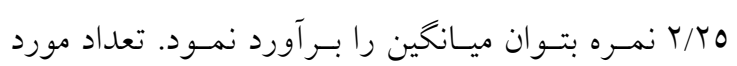

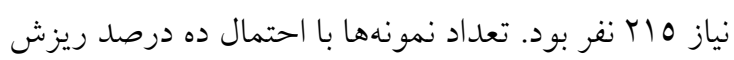

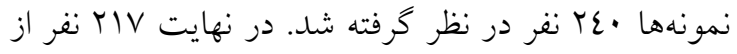
جامعه يُوهش، يرسشنامهها را به طور كامل تكميل نمودند.

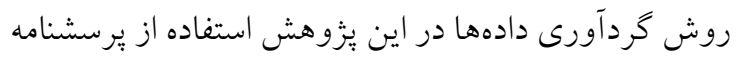

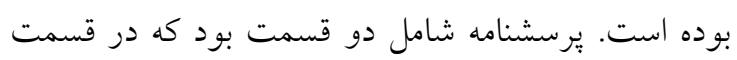
اول مشخصات جمعيت شناختى شركت كنندها از قبيل

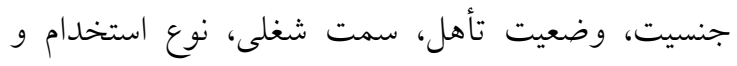


(\%Vع/V)

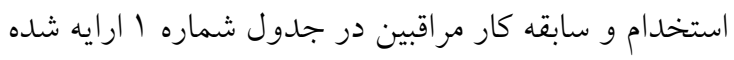

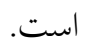

جدول شماره ا: توزيع فراوانى جنسيت، وضعيت تاهل، سمت شغلى، نوع استخدام و سابقه كار مراقبين

\begin{tabular}{|c|c|c|c|}
\hline درصد & تعداد & & متغير \\
\hline$r V / r$ & 11 & مرد & جنسيت \\
\hline$T r / V$ & Irr & زن ان & \\
\hline ro/r & 00 & مجرد & وضعيت \\
\hline$V \varepsilon / V$ & $17 r$ & متأهل & تأهل \\
\hline $1 T / 9$ & $r \Lambda$ & كاردان اتاق عمل & \\
\hline$r T / l$ & $\varepsilon \wedge$ & كارشناس اتاق عمل & \\
\hline$V / \varepsilon$ & 17 & يُرستار اتاق عمل & \\
\hline$\varepsilon / 7$ & $1 \cdot$ & كاردان هوشبرى & سمت \\
\hline $19 / 1$ & $\varepsilon r$ & كارشناس هوشبرى & ا شغلى \\
\hline $1 \cdot / r$ & $r r$ & متخصص بيهوشى & \\
\hline $1 \cdot / 7$ & $r$ & متخصص جراحى & \\
\hline $\mid r / \varepsilon$ & rV & دستيار جراحى & \\
\hline YN/I & 71 & طرحى & \\
\hline $9 / N$ & rl & 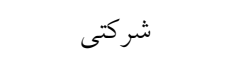 & \\
\hline$\Lambda / V$ & 19 & تبصرهاى & نوع \\
\hline$T / 0$ & $1 \varepsilon$ & قراردادى & استخدام \\
\hline $0 / 0$ & Ir & بيمانى & \\
\hline$\varepsilon 1 / 0$ & 9. & رسمى & \\
\hline$r \tau / \varepsilon$ & va & زير ه سال & \\
\hline $19 / 1$ & $\varepsilon r$ & 0 & \\
\hline $\mid r / \Lambda$ & $r \cdot$ & 1ا تا 10 سال & ل سبقه كار \\
\hline $1 \cdot / 7$ & $r$ & T17 تا •r سال & \\
\hline $19 / 2$ & $\varepsilon r$ & بالاى •r سال & \\
\hline
\end{tabular}

ميانخين نمره كل نخرش ايمنى مراقبين سلامت 7 • 09 با

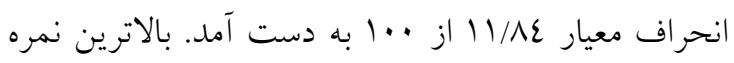
مربوط به بعد شناخت استرس با ميانخين سو لهو و كمترين

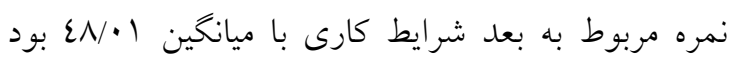

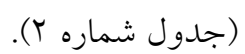

مسئولين مربوطه در مراكز آموزشى درمانى منتخب، وارد

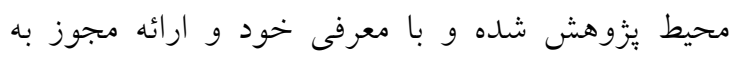

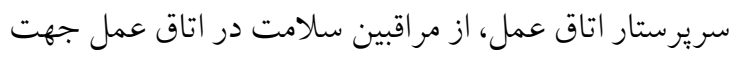

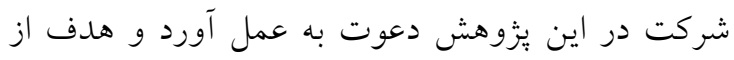

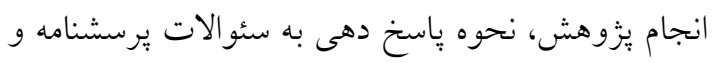

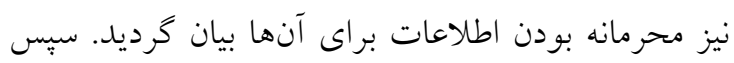

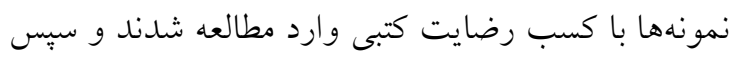

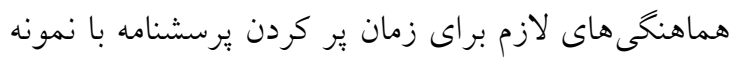

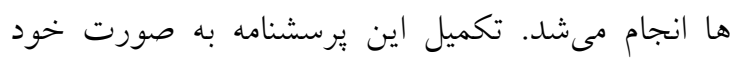

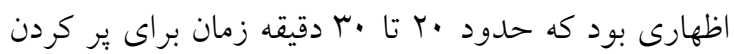

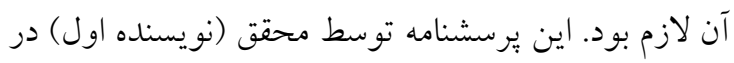
ابتداى شيفت به كاركنان داده شد تا قبل از شروع اولين بردين

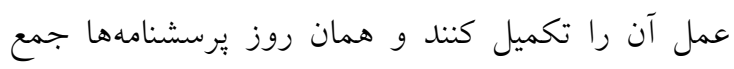

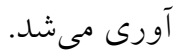
اطلاعات جمع آورى شده با استفاده از نرم افزار كPSS

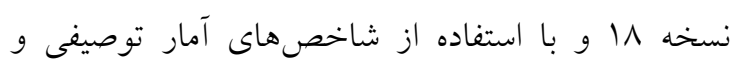

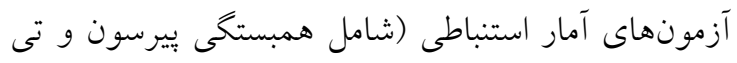

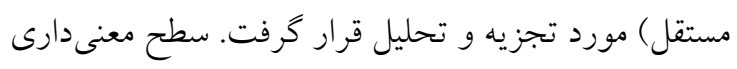

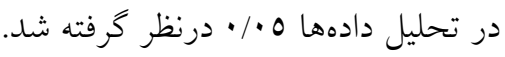

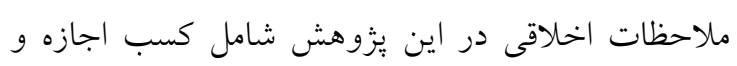

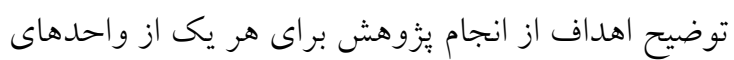

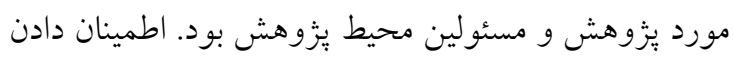

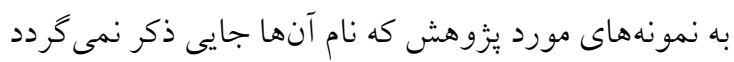

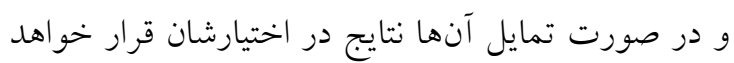

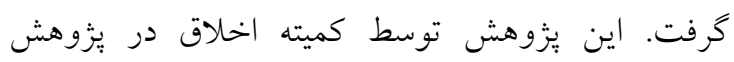

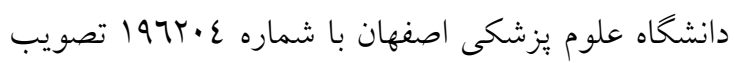

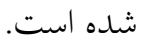

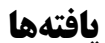

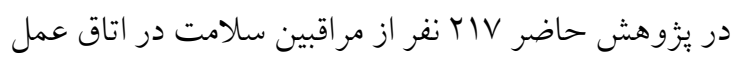

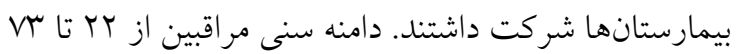

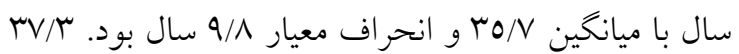

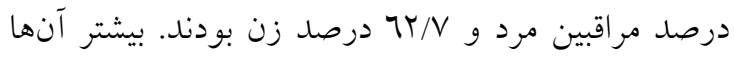


جدول شماره r: ميانكين نمره نكَرش ايمنى و ابعاد آن (از + + (1) در مراقبين سلامت

\begin{tabular}{|c|c|c|c|c|}
\hline حداكثر & حداقل & انحر اف معيار & ميانخين & ابعاد اد \\
\hline $9 \varepsilon / 10$ & $\mid \Lambda / \mu \Lambda$ & $11 / \wedge \varepsilon$ & $09 / \cdot 7$ & نمره كل نخرش ايمنى \\
\hline $1 \cdots$ & $r \cdot / \Lambda r$ & $10 / 2 \varepsilon$ & $7 Y / \cdot 7$ & جو كار تيمى \\
\hline $97 / \varepsilon r$ & $1 \cdot / N 1$ & $1 \varepsilon / 19$ & $O V / Y T$ & جو ايمنى \\
\hline $1 \cdots$ & • & $r \cdot 10$. & $7 \cdot / 9 \varepsilon$ & رضايت شغلى \\
\hline $1 \cdots$ & ro & $\mid V / \varepsilon 7$ & $\vee q / q \mu$ & شناخت استرس \\
\hline $9 Y / \Lambda T$ & $V / I \varepsilon$ & $17 / / \%$ & $O r / 91$ & مديريت \\
\hline $1 \cdots$ & 0 & $1 \Lambda / \cdot V$ & $\{\Lambda / \cdot 1$ & شرايط كارى \\
\hline
\end{tabular}

ضريب همبستكى بيرسون نشان داد كه نمره شناخت استرس با سن (q// • P=) رابطه معنى دار نداشت، اما نمره كل نخرش ايمنى و ساير ابعاد آن با سن رابطه مستقيم

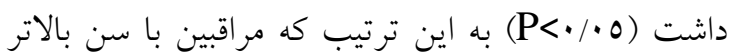
ميانخين نمره نخرش ايمنى بالاترى داشتند. هم جنين
ضريب همبستكى نشان داد كه نمره شناخت استرس با

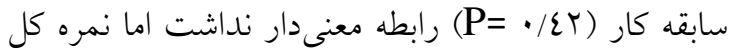
نخرش ايمنى و ساير ابعاد آن با سابقه كار رابطه مستقيم

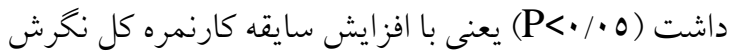
ايمنى در مراقبين بالاتر بود (جدول شماره س).

جدول شماره ऍّ: ضرايب همبستكى بين نمره كل نغَرش ايمنى و ابعاد آن با سن و سابقه كار

\begin{tabular}{|c|c|c|c|c|}
\hline \multicolumn{2}{|c|}{ سابقه كار } & \multicolumn{2}{|c|}{ سن سن } & \multirow[b]{2}{*}{ ابعاد اد } \\
\hline P-value & $\mathrm{r}$ & P-value & $\mathrm{R}$ & \\
\hline$<\bullet / \cdots 1$ & רזוא/ & $<\cdot / \bullet \cdot$ & $\cdot / r \vee \varepsilon$ & نمره كل نخرش ايمنى \\
\hline$\cdot / \cdots \varepsilon$ &.$/ 19 \varepsilon$ & $<\cdot / \bullet \cdot$ & $\cdot / r 79$ & جو كار تيمى \\
\hline$\cdot / \cdot r$ &.$/ 10$. & $\cdot / \cdot r$ & . /10r & جو ايمنى \\
\hline.$\cdots 1$ & $\cdot / T Y I$ & $<\cdot / \bullet \cdot$ & $\cdot / \pi q \varepsilon$ & رضايت شغلى \\
\hline$\cdot / \varepsilon r$ & .1 .00 & $\cdot \pi q$ &.$/ \cdot 7 r$ & شناخت استرس \\
\hline.$/ \cdots 1$ & $\cdot / 417$ & $<\cdot / \bullet \cdot$ & $\cdot r \varepsilon v$ & مديريت \\
\hline .1 .20 &.$/ 117$ & 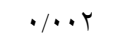 & $\cdot / T Y I$ & شرايط كارى \\
\hline
\end{tabular}

ايمنى (

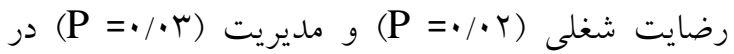
مراقبين متأهل به طور معنىدارى بيشتر از مراقبين مجرد بود اما ميانخين ساير ابعاد نخرش ايمنى بين مراقبين متأهل و مجرد اختلاف معنىدار نداشت (P > P (جدول

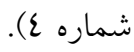

آزمون تى مستقل نشان داد كه ميانخين نمره كل نخرش

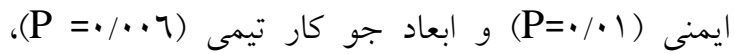

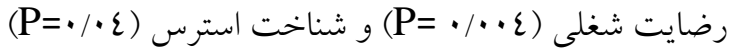
در گروه مردان به طور معنىدارى بيشتر از زنان بود اما ميانخين ساير ابعاد نخرش ايمنى بين زنان و مردان اختلاف معنى دار نداشت (0 • P P). ميانخين نمره كل نخرش 


\begin{tabular}{|c|c|c|c|c|c|c|}
\hline P-value & $\mathbf{t}$ & df & انحر اف معيار & ميانغين & & ابعاد \\
\hline & & & $\mid T / T \varepsilon$ & $71 / V \varepsilon$ & 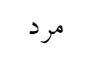 & نمره كل نخرش ايمنى \\
\hline \multirow[t]{2}{*}{$\cdot / \cdot 1$} & $r / 7$. & rio & $11 / r \varepsilon$ & $O V / \varepsilon V$ & زن ان & \\
\hline & & & 1r/or & $70 / v 9$ & 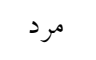 & جو كار تيمى \\
\hline \multirow[t]{2}{*}{$\cdot / \cdot 7$} & $r / v q$ & Y10 & $17 / 11$ & $09 / \wedge r$ & ز - ان & \\
\hline & & & $1 \varepsilon / \varepsilon r$ & $O N / \Sigma Y$ & 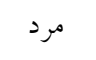 & جو ايمنى \\
\hline \multirow[t]{2}{*}{$\cdot / \mu_{\Lambda}$} & $\cdot / \Lambda \Lambda$ & Y10 & $10 / 1 \mathrm{~V}$ & $07 / 0 \mathrm{~V}$ & 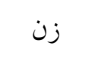 & \\
\hline & & & TI/TO & $77 / \cdot 0$ & 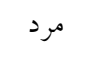 & رضايت شغلى \\
\hline \multirow[t]{2}{*}{$\cdot / \cdot \varepsilon$} & $r / \Lambda \Lambda$ & Y10 & $19 / 2 V$ & $O V / 9$. & زن زن & \\
\hline & & & $19 / .1$ & $\Lambda r / T \varepsilon$ & مرد & شناخت استرس \\
\hline \multirow[t]{2}{*}{$\cdot / \cdot \varepsilon$} & $1 / V V$ & Y10 & $17 / \pi$ & $V \wedge / \mu I$ & زن & \\
\hline & & & $1 V / \varepsilon \varepsilon$ & $0 \varepsilon / 09$ & 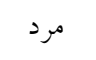 & مديريت \\
\hline \multirow[t]{2}{*}{$\cdot /$ To } & $1 / 17$ & Y10 & $10 / 70$ & $01 / 9 r$ & 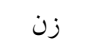 & \\
\hline & & & $\mid \Lambda / \varepsilon \wedge$ & 0.129 & مرد & شرايط كارى \\
\hline \multirow[t]{2}{*}{$\cdot / 14$} & $1 / 07$ & Y10 & IV/NT & $\varepsilon 7 / 0 \varepsilon$ & زن & \\
\hline & & & $11 / \wedge \varepsilon$ & $07 / \% 0$ & مجرد & نمره كل نخرش ايمنى \\
\hline \multirow[t]{2}{*}{$\cdot / \cdot \varepsilon q$} & $-1 / 9 V$ & Ylo & $11 / v \varepsilon$ & $09 / 91$ & 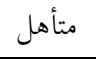 & \\
\hline & & & 17/7r & $09 / \cdot 1$ & مجرد & جو كار تيمى \\
\hline \multirow[t]{2}{*}{$1 / 20$} & $-1 / v$ & Y10 & $1 \varepsilon / 99$ & $7 \pi / .9$ & متأهل & \\
\hline & & & $10 / 1$ & $07 / \cdot \varepsilon$ & مجرد & جو ايمنى \\
\hline \multirow[t]{2}{*}{$\cdot / 2 \Lambda$} & $-\cdot / v \cdot$ & rio & $1 \varepsilon / \wedge \varepsilon$ & $O V / T V$ & متأهل & \\
\hline & & & 19/Ar & $00 / 20$ & مجرد & رضايت شغلى \\
\hline \multirow[t]{2}{*}{$\cdot / \cdot r$} & $-T / \mu r$ & r10 & $r \cdot / \varepsilon \varepsilon$ & $7 r / \Lambda 1$ & 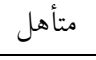 & \\
\hline & & & $10 / \varepsilon \varepsilon$ & $\Lambda \cdot / 0 \mathrm{~V}$ & مجرد & شناخت استرس \\
\hline \multirow[t]{2}{*}{./vo } & $\cdot / \mu_{1}$ & Y10 & $\mid N / 1 \varepsilon$ & Va/vi & متأهل & \\
\hline & & & $17 / 9 r$ & $\varepsilon \Lambda / \wedge r$ & مجرد & مديريت \\
\hline \multirow[t]{2}{*}{$\cdot / \cdot r$} & $-\Gamma / 17$ & r10 & $10 / 9 \mathrm{~V}$ & $0 \varepsilon \pi$. & متأهل & \\
\hline & & & IV/A. & $\varepsilon 0 / 7 \varepsilon$ & مجرد & شرايط كارى \\
\hline$\cdot / r 7$ & $-1 / 14$ & Y10 & $11 / 10$ & $\varepsilon \wedge / \wedge \mu$ & متأهل & \\
\hline
\end{tabular}

جدول شماره 0: توزيع فراوانى نمره دهى نكَرش مراقبين سلامت از نظر رتبه بندى وضعيت ايمنى بيمار

\begin{tabular}{|c|c|c|}
\hline درصد & فراوانى & نمره كل نخرش ايمنى \\
\hline$r / \mathrm{V}$ & $\wedge$ & عالى \\
\hline$\varepsilon 0 / 7$ & 99 & بسيار خوب \\
\hline$\varepsilon Y / \varepsilon$ & 94 & متوسط \\
\hline $\mathrm{V} / \Lambda$ & IV & نامناسب \\
\hline$\cdot 10$ & 1 & ضعيف \\
\hline
\end{tabular}

جدول شماره 0 توزيع فراوانى نمره دهى نخرش افر اد مورد

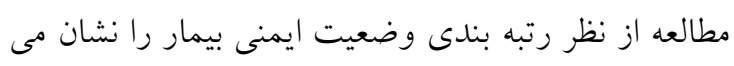

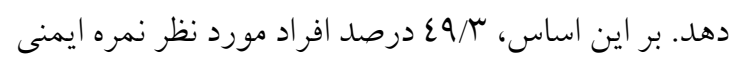
بيمار را بسيار خوب و عالى توصيف كردند. اخرجهـ V/

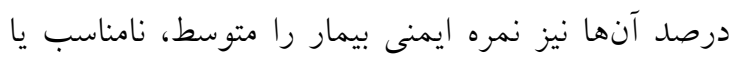
ضعيف ذكر كردند. 
يافتها با نتايج مطالعه تورانى و همكاران (1) و مطالعه عرب

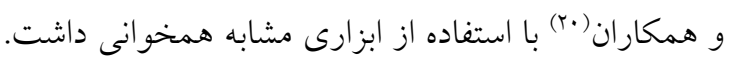

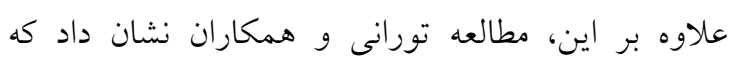

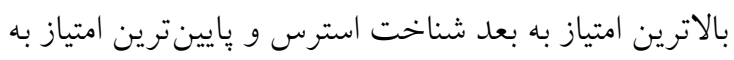

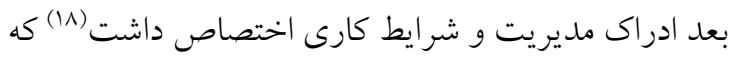
يافتهاى مطالعه حاضر را تأييد مىكند. در مطالعه

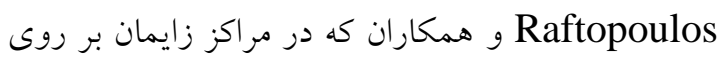
ماماها انجام شده است، بالاترين امتياز به ترتيب به ابعاد كار كروهى، جو ايمنى، رضايت شغلى و شرايط كارى

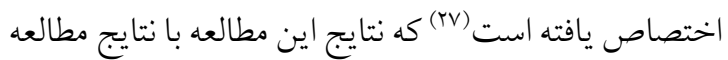

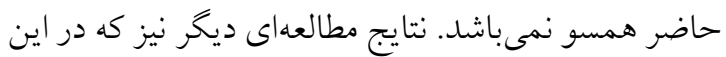
حوزه در بخشهاى مراقبت ويزه در استراليا انجام شد، نشان داد بيشترين امتياز براى كار گروهى و كمترين امتياز

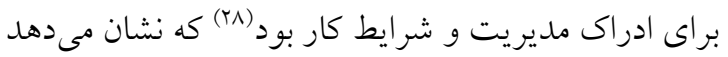

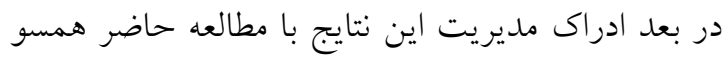

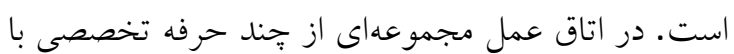

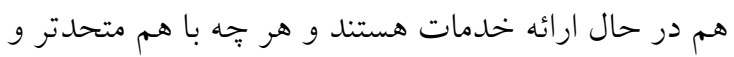

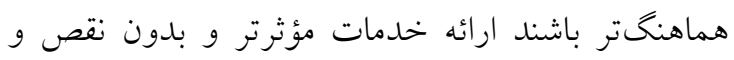

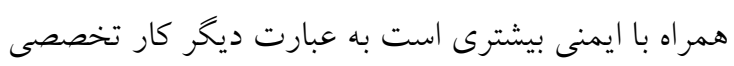

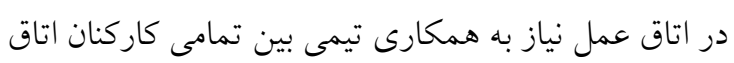

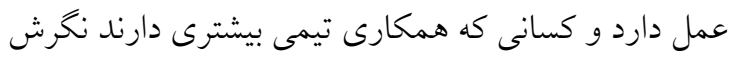
ايمنى بالاترى نسبت به بيمار دارند.

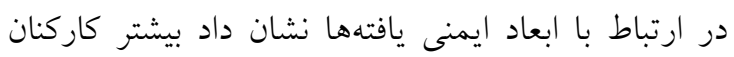

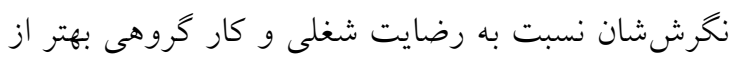

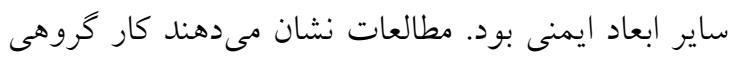
و نغرش ايمنى مىتواند بر ايمنى مراقبت از بيمار تأثير

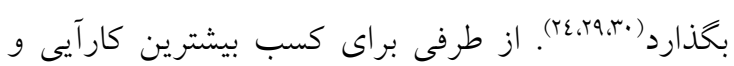
اثربخشى در هر سازمانى، كاركنان بايد علاوه بر داثتن لختئ تخصص و مهارت، عشق و علاقه به كار خود داشته باشند (r)مagusa اشاره مى كند بدون اين خصوصنات هيج كس نمىتواند در اتاق عمل كار آيى و اثربخشى بالايى ائس داشته باشد ("r). بنابراين مراقبى كه تعهد كارى به شغل و و سازمان خود دارد نخرش بهترى نسبت به ايمنى بيمار دارد

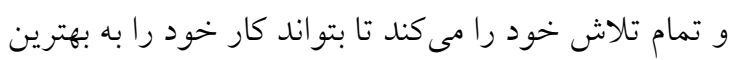

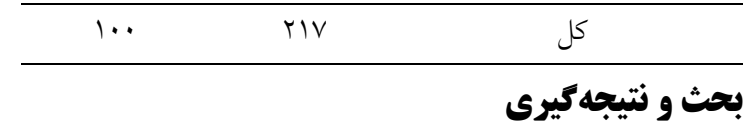

مطالعه حاضر به بررسى نغرش مرى مراقبين سلامت به ايمنى

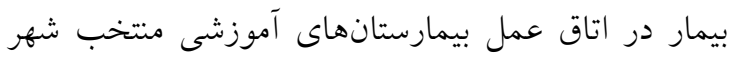
اصفهان يرداخته است. در مطالعه حاضر همبستخى بين نمره كل نخرش ايمنى و ابعاد آن با جنسيت، سن و سابقه كار مورد بررسى قرار

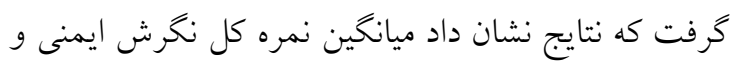

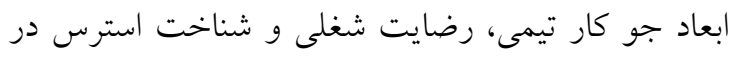

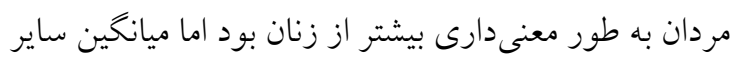

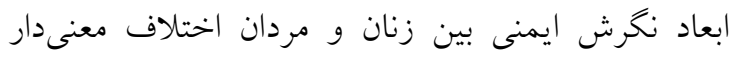

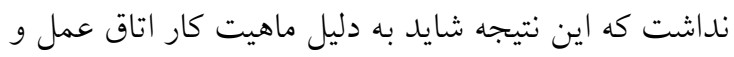

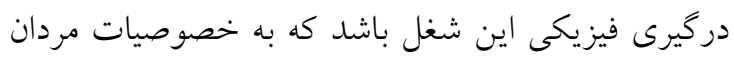
كه بيشتر به كارهاى فيزيكى تمايل دارند، شبيهتر است.

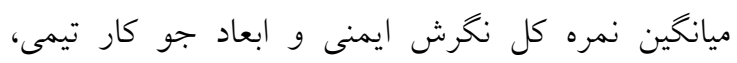
رضايت شغلى و مديريت در مطالعه حاضر در مراقبين

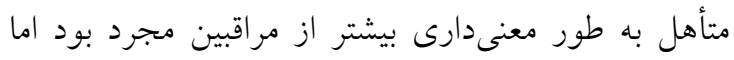

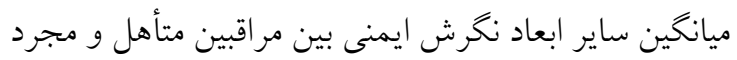

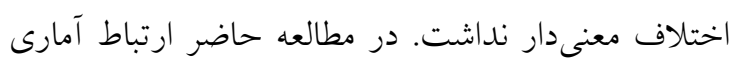

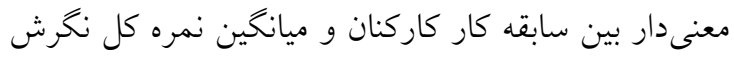
ايمنى مشاهده شد يعنى با افزايش سايقه كار، نمره كل

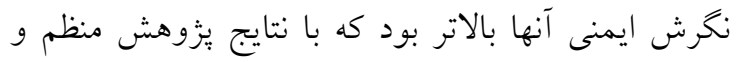
سلطانزاده كه نشان دادند كاركران با افزايش سن و سابقه، احتمال خطا و تصادف بيشتر و ميانخين نمره نخرش ايمنى

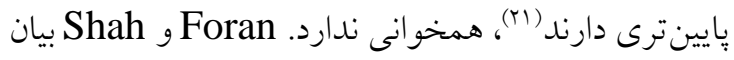

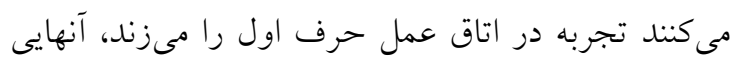

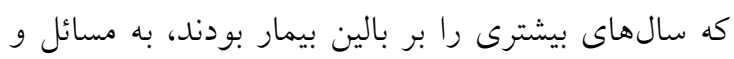

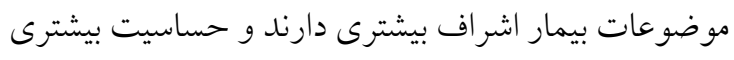
نسبت به ايمنى بيمار و ييشخيرى از خطاهايى كه شايد قبلاً

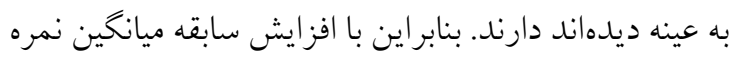
نخرش ايمنى بالاترى دارند (ro.r7). بر اساس يافتهها حدود نيمى از مراقبين سلامت در اتاق باق بات

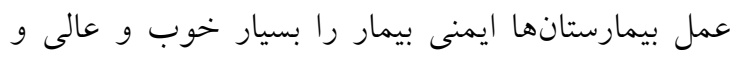

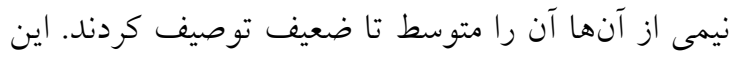


هاى نظارتى و كنترلى از طرف مسئولين صورت يذيرد.

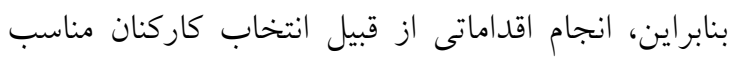

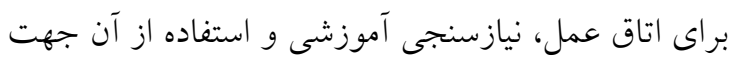

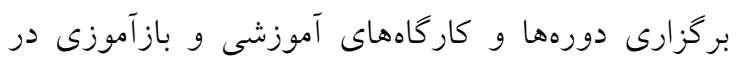

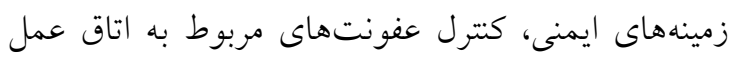

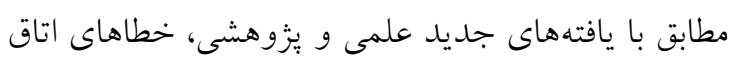

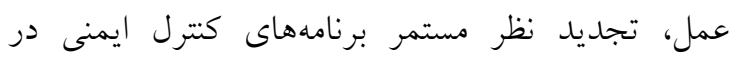
بيمارستان، برآورد هزينههاى ناشى از عدم رعايت ايمنى و

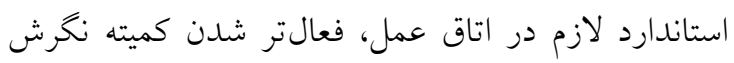
ايمنى و آموزشهاى درون بخشى در اتاق عمل با توجه به دمانه

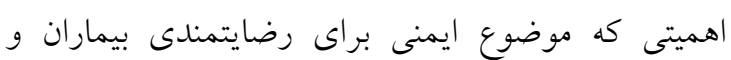
كاركنان دارد بسيار مفيد بوده و بهتر است توسط مسئولان بيمارستانها مد نظر قرار كيرد.

تعارض منافع: هيجگگ نه تعارض منافع از سوى نويسندگان

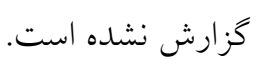

\section{تقدير و تشكر}

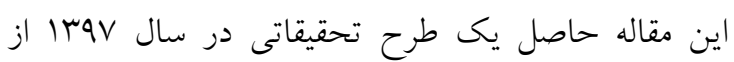

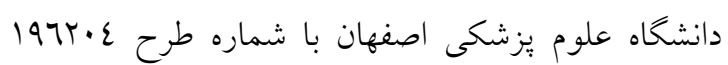

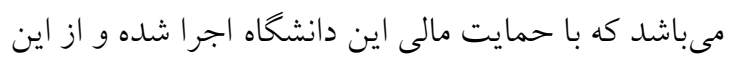

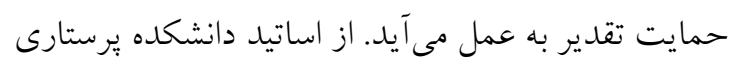

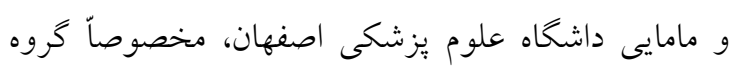

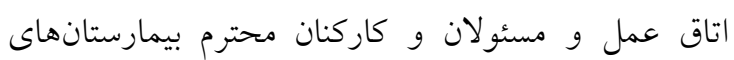
آموزشى منتخب شهر اصفهان شامل بيمارستانهاى الزهرا،

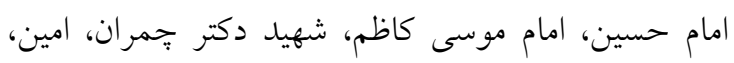

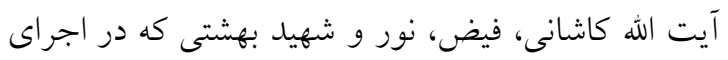

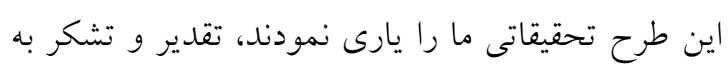
عمل مى آيد.
نحو انجام دهد تا هيج مشكل اضافى براى بيمار ايجاد نشود و سلامت و بهبودى بيمار خود را تسريع كند.

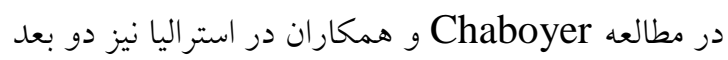

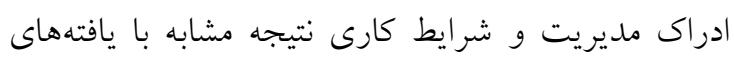

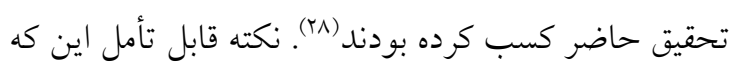
هر جهه مديران بيمارستان و مديريت هر كدام از حرفهاى

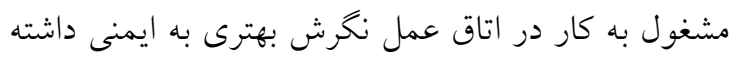

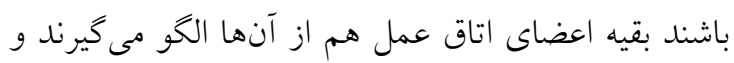
شرايط كارى به سمت ارتقاى ايمنى بيمار بيش مى بـرود.

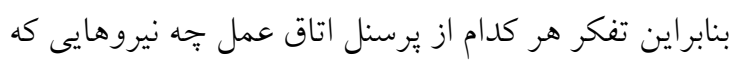
مستقيم با بيمار در تماس هستند و جهه نيروهايى كه يشتيبان اين نيروها هستند در ايمنى بيمار نقش به سزايى دارد.

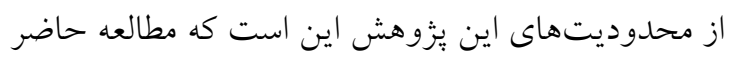

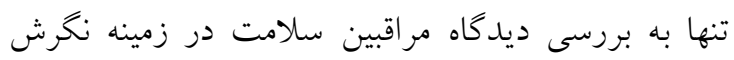
ايمنى بيمار در بيمارستانهاى دولتى برداخته است و امكان

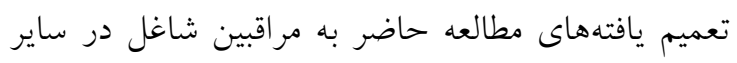

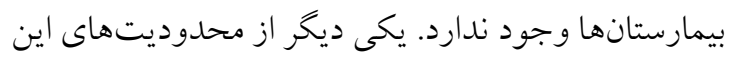
مطالعه، جمع آورى اطلاعات از طريق خودارزيابى نمونهها

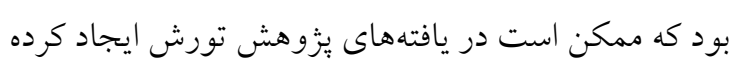
باشد.

در بززوهش حاضر مشخص كرديد كه ميانخين نخرش ايمنى بانس در ميان همه كاركنان اتاق عمل ايده آل به نظر نمىرسيد.

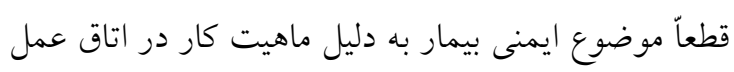

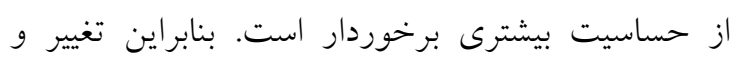

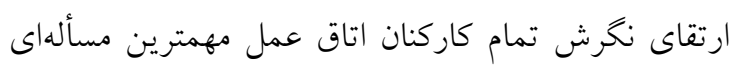
است كه بايد در اتاقهاى عمل مورد توجه ويزه قرار خيرد.

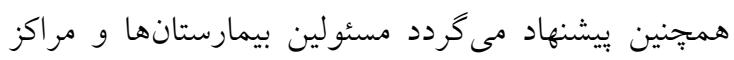

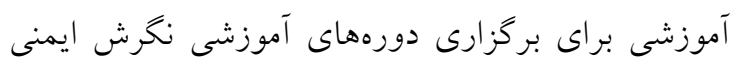

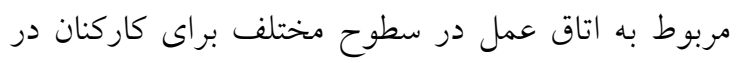
بيمارستانها برنامهريزى كرده و ييخيرى جلدى و ايجاد اهرم

\section{References:}

1. Carayon P. Handbook of human factors and ergonomics in health care and patient safety. CRC Press; 2006 Sep 8.

2. Gandhi TK, Kaplan GS, Leape L, Berwick DM, Edgman-Levitan S, Edmondson A, Meyer GS, Michaels D, Morath JM, Vincent C, Wachter R. Transforming concepts in patient safety: a progress report. BMJ Qual Saf. 2018;27(12):1019-26. 
3. Ingvarsdottir E, Halldorsdottir S. Enhancing patient safety in the operating theatre: from the perspective of experienced operating theatre nurses. Scand J Caring Sci. 2018 Jun;32(2):951-60.

4. Kalra JJ, Kopargaonkar A. Quality Care and Patient Safety: Strategies to Disclose Medical Errors. InInternational Conference on Applied Human Factors and Ergonomics 2017 (159-67).

5. Colla JB, Bracken AC, Kinney LM, Weeks WB. Measuring patient safety climate: a review of surveys. BMJ Quality \& Safety. 2005;14(5):364-6.

6. Brasaite I, Kaunonen M, Suominen T. Healthcare professionals' knowledge, attitudes and skills regarding patient safety: a systematic literature review. S Scand J Caring Sci. 2015;29(1):30-50.

7. Walpola RL, Fois RA, McLachlan AJ, Chen TF. Enhancing generational change in patient safety attitudes through peer leadership. BMJ Simul Technol Enhanc Learn. 2016 Sep 1;2(3):87-9.

8. Berry JC, Davis JT, Bartman T, Hafer CC, Lieb LM, Khan N, Brilli RJ. Improved Safety Culture and Teamwork Climate Are Associated With Decreases in Patient Harm and Hospital Mortality Across a Hospital System. J Patient Saf. 2016(7).

9. Akbari Sari A, Doshmangir L. An overview of methods for identifying and measuring adverse events in health care and their strengths and weaknesses. Journal of Iranian Scientific Hospital Association. 2009;32:51-6. [Persian]

10. Famolaro T, Yount ND, Burns W, Flashner E, Liu H, Sorra J. Hospital survey on patient safety culture: 2016 user comparative database report. Agency for Healthcare Research and Quality; 2016.

11. Weaver SJ, Lubomksi LH, Wilson RF, Pfoh ER, Martinez KA, Dy SM. Promoting a culture of safety as a patient safety strategy: a systematic review. Annals of internal medicine. 2013;158(5_Part_2):369-74.

12. DiCuccio MH. The relationship between patient safety culture and patient outcomes: a systematic review. J Patient Saf. 2015;11(3):135-42.

13. Waterson P. Patient Safety Culture and Organisational Behaviour. Patient Safety Culture: CRC Press; 2017. P:70-93.

14. Zakari NM. Attitude of academic ambulatory nurses toward patient safety culture in Saudi Arabia. Life Science Journal. 2011;8(3):230-7.

15. Agnew Ç, Flin R. Safety Culture in Practice: Assessment, Evaluation, and Feedback. Patient Safety Culture: Theory, Methods and Application. 2018 ;110:235-56.

16. Hall LH, Johnson J, Watt I, Tsipa A, O'Connor DB. Healthcare staff wellbeing, burnout, and patient safety: a systematic review. PloS one. 2016;11(7):e0159015.

17. Geraghty A, Renwick A, Yalamarthi S, McIlhenny C. Patient safety attitudes in core surgical trainees. International Journal of Surgery. 2015;1(23):S105.

18. Tourani S, Khodayari Zarnaq R, Arabloo J, Esmaili A, Taghizadeh S, Khosravizadeh O. A survey on patient safety using the farsi version of the safety attitudes questionnaire in Iran. Journal of Payavard Salamat. 2016;10(1):82-92.

19. Khalilzadeh H, Zare Fazlollahi Z, Mohaddesi H, Hemmati Maslak Pak M. Attitude among urmia medical university health workers about patient safety. The Journal of Urmia Nursing and Midwifery Faculty. 2013;11(8):606-13. [Persian]

20. Arab M, Mohammadian F, Rahmani A, Rahimi A, Omidi L, Asghari M. Safety Attitude in Operating Room's Staff's in selected hospitals of Tehran University of Medical Sciences in 2013. Journal of Hospital. 2014;13(3):25-33 [Persian]

21. Monazzam MR, Soltanzadeh A. The relationship between the worker's safety attitude and the registered accidents. Journal of research in health sciences. 2009;9(1):17-20. [Persian]

22. Tourani S, Khodayari Zarnaq R, Arabloo J, Esmaili A, Taghizadeh S, Khosravizadeh O. A survey on patient safety using the farsi version of the safety attitudes questionnaire in Iran. Journal of Payavard Salamat. 2016;10(1):82-92. [Persian]

23. Mousavi SM, Dargahi HO, Hasibi ME, Mokhtari ZA, Shaham GO. Evaluation of safety standards in operating rooms of Tehran University of Medical Sciences (TUMS) Hospitals in 2010. Journal of Payavard Salamat. 2011;5(2):10-7.

24. Sexton JB, Helmreich RL, Neilands TB, Rowan K, Vella K, Boyden J, Roberts PR, Thomas EJ. The Safety Attitudes Questionnaire: psychometric properties, benchmarking data, and emerging research. BMC Health Serv Res. 2006;6(1):44. 
25. Foran P. Undergraduate surgical nursing preparation and guided operating room experience: A quantitative analysis. Nurse education in practice. 2016;16(1):217-24.

26. Shah MM, Hunter BW, Sweeney JF, Lin E, Perez SD, Parker C, Davis Jr SS. Operating Room Efficiency in Bariatric Surgery: The Effect of Team Member Experience on Operative Times in Laparoscopic Roux-en-Y Gastric Bypass. Bariatr Surg Prac Patient Care. 2017;12(3):100-6.

27. Raftopoulos V, Savva N, Papadopoulou M. Safety culture in the maternity units: a census survey using the Safety Attitudes Questionnaire. BMC Health Serv Res. 2011;11(1):238.

28. Chaboyer W, Chamberlain D, Hewson-Conroy K, Grealy B, Elderkin T, Brittin M, McCutcheon C, Longbottom P, Thalib L. CNE article: safety culture in Australian intensive care units: establishing a baseline for quality improvement. Am J Crit Care. 2013;22(2):93-102.

29. Pinheiro JP, de Sousa Uva A. Safety climate in the operating room: Translation, validation and application of the Safety Attitudes Questionnaire. Revista portuguesa de saúde pública. 2016;34(2):107-16.

30. Erestam S, Haglind E, Bock D, Andersson AE, Angenete E. Changes in safety climate and teamwork in the operating room after implementation of a revised WHO checklist: a prospective interventional study. Patient safety in surgery. 2017;11(1):4.

31. Ragusa PS, Bitterman A, Auerbach B, Healy III WA. Effectiveness of surgical safety checklists in improving patient safety. Orthopedics. 2016;39(2):e307-10. 